Anuario del Instituto de Historia Argentina, vol. 17, nº 2, e056, diciembre 2017.

ISSN 2314-257X

Universidad Nacional de La Plata.

Facultad de Humanidades y Ciencias de la Educación.

Centro de Historia Argentina y Americana

\title{
"Atrás de cada pibe de la calle hay un padre desocupado". Organizaciones sociales y sindicales en lucha por la infancia digna (1983 - 2001)
}

\author{
"Behind every street boy there is an unemployed parent". Social \\ organizations and trade unions fighting for a decent childhood \\ (1983 - 2001)
}

\section{Paula Nurit Shabel}

Universidad de Buenos Aires - CONICET, Argentina | gonza_rubio@hotmail.com

\section{PALABRAS CLAVE RESUMEN}

Niñez El objetivo del presente artículo es analizar la relación entre el desarrollo de las organizaciones sociales que trabajaron con la infancia pobre y la situación del movimiento obrero organizado entre 1983 y 2001.

Movimiento obrero

Organizaciones sociales Estudiamos el período a partir de una revisión bibliográfica y de material periodístico que ponemos en relación con entrevistas realizadas a distintos protagonistas y material audiovisual producido por ellos y sus instituciones. Como resultado, ubicamos una articulación entre organizaciones y sindicatos que fortaleció ambas luchas y profundizó su legitimidad, ensanchando el propio concepto de clase y de infancia.

\section{KEYWORDS ABSTRACT}

Childhood The aim of this paper is to analyze the relationship between the social organizations that work with the Trade unions childhood of the poor and the trade unions during 1983 - 2001. We have made a bibliographic and journalistic revision to study the period and then crossed it with interviews made to the people involved in Social organizations these processes at that time, as well as with audiovisual material produces by them and their institutions. As a result, we established a linkage between organizations and trade unions that strengthen their claims and bolster their legitimacy, enlarging the concept of class and childhood.

Recibido: 25 de abril de 2017 | Aceptado: 25 de septiembre de 2017 | Publicado: 15 de diciembre de 2017

Cita sugerida: Shabel, P. (2017). “Atrás de cada pibe de la calle hay un padre desocupado”. Organizaciones sociales y sindicales en lucha por la infancia digna (1983 - 2001). Anuario del Instituto de Historia Argentina, 17(2), e056. https://doi.org/10.24215/2314257Xe056 


\section{Introducción}

En los 80 y al calor de las luchas por la recuperación democrática se gestó un movimiento por los derechos de la niñez, tanto en Argentina como en otras partes del continente, que se alejaba de los discursos liberales y se nutría de una perspectiva clasista en la que se afirmaba que la lucha por la niñez digna y por la igualdad social eran parte del mismo proceso, y que el silenciamiento de esta generación era una desigualdad más producida por el capitalismo (Liebel, 2006). En el país, muchas organizaciones sociales y políticas llevaron esta querella como bandera y juntas se constituyeron en el Movimiento Nacional Chicos del Pueblo (MNCP), que se relacionó rápidamente con el movimiento obrero organizado en la Confederación General del Trabajo (CGT) y luego formó parte del proyecto de la Central de Trabajadores de la Argentina (CTA), en una apuesta por la articulación de las problemáticas de una clase proletaria en transformación donde cabían desempleados, movimientos sociales, mujeres, jubilados y niños y niñas.

En este marco, el objetivo del presente trabajo es analizar la relación entre el desarrollo de las organizaciones de niñez y la situación del movimiento obrero organizado, asumiendo que es en el seno de la lucha de clases donde se producen los avances y retrocesos en materia de infancia. Como hipótesis, sostenemos, entonces, que la articulación entre estos dos colectivos fortaleció ambas luchas y profundizó su legitimidad. El período que abordaremos abarca desde la recuperación democrática en la Argentina en 1983 hasta el 2001, año en que el MNCP realizó su primera marcha nacional, abriendo un nuevo período en la historia de esta problemática particular, para la cual el presente trabajo es un primer acercamiento.

Utilizamos el concepto de infancia/niñez digna para referirnos a una infancia que pueda ejercer la totalidad de sus derechos, incluyendo la participación en los procesos de lucha para su conquista. Esta definición es tomada de los discursos de los protagonistas del proceso analizado, que sostienen, principalmente, que nada de esto es posible sin una transformación radical en la distribución de la riqueza: “una infancia digna en una sociedad justa” (Omar Giuliani, comunicación personal, 23 de noviembre de 2015 y 04 de abril de 2016ํㅗ); y “no habrá cambio en el capitalismo (...) la única manera de lograr una sociedad distinta es a través del socialismo. No existe un capitalismo más humano, el capitalismo fabrica la pobreza y los pibes en la calle” (Morlachetti en CTA, 2013).

Estas organizaciones también afirman que es necesario ubicar a niños y niñas como protagonistas de los procesos de lucha de la clase, entendiendo que son también ellos y ellas las afectadas por la explotación, y que tienen la capacidad de comprender y actuar para transformar esa realidad. Entendemos, por todo esto, que la infancia es una construcción política y social siempre anclada en su contexto histórico, y siempre disputada por diversos sectores que depositan en ella valores y obligaciones que responden a sus intereses más amplios (Carli, 2012). Al mismo tiempo, utilizamos esta categoría, así como la de adolescentes y jóvenes (indistintamente) para referirnos a aquellas personas que tienen hasta 18 años, sin desconocer las discusiones que suscitan estas clasificaciones ni la arbitrariedad de dicho límite etario, pero que aceptamos como noción hegemónica que rige leyes, discursos y prácticas de la realidad que estudiamos.

Consideramos fundamental sistematizar este recorrido, tanto para visibilizar el rol del campo popular en la lucha por los derechos de los niños como para valorizar la perspectiva que este sector social tiene sobre la problemática. Sin ser objeto del presente análisis, reconocemos sin embargo, 
que no fue solo la clase obrera organizada la que avanzó en este sentido, sino que también influyeron las presiones sociales provenientes de sectores medios, referentes políticos varios, la iglesia y organismos internacionales como UNICEF. En función de nuestros objetivos, organizamos el trabajo en dos períodos históricos, que si bien son un tanto arbitrarios como cualquier periodización (Cucuzza, 1996), no son azarosos, ya que reflejan distintos momentos de la lucha popular por la infancia digna, en términos de organización interna y articulación con otras disputas sociales y políticas. Un primer momento, de 1983 hasta 1990, en el que se gesta la problemática específica de la infancia pobre en la sociedad argentina, y un segundo período, de 1990 hasta 2001, en el que dicha problemática se radicaliza en el marco de nuevas estrategias de lucha de la clase trabajadora.

\section{Algunas cuestiones teóricas y metodológicas}

El presente trabajo se inscribe en una investigación doctoral más amplia sobre las organizaciones sociales de niños y niñas en la Argentina contemporánea. El marco teórico establecido se desprende de una serie de lecturas realizadas sobre el campo de los estudios de infancia que, si bien es reciente, ya ha producido numerosos trabajos desde las distintas ciencias sociales. Nuestro incipiente aporte es por la visibilización de las organizaciones sociales en el complejo entramado de la niñez, y por eso valoramos los análisis hechos desde la antropología en cuanto a la producción de infancia desde el Estado (Barna, 2013; Villalta, 2010) y en relación con la sociedad civil (Santillán, 2012). Además, nos apoyamos en las investigaciones de Gentile (2011), que se preguntó por la construcción de la niñez en organizaciones sociales, al igual que Carli (2012) desde la pedagogía y Cosse (2006) y Ciafardo (1992) desde la historia, tal como Camarero (2007; Camarero \& Herrera, 2005), que en su historia de los partidos políticos aporta elementos en esta dirección.

En términos metodológicos, realizamos una serie de entrevistas a los protagonistas del proceso, referentes de organizaciones de niñez y del movimiento obrero, particularmente a aquellos dirigentes que más se interesaron y ocuparon por las problemáticas de infancia, según lo muestran los videos conseguidos y los relatos recopilados. Las mismas fueron tomadas en forma individual en los lugares de trabajo de los diferentes actores (organización, sindicato, local partidario, etc.), a los que accedimos como investigadoras y también militantes del campo popular con la intención de dar luz a estas historias, desde una investigación comprometida (Fals Borda, 1990; Sirvent, 2008). Además, utilizamos materiales escritos y audiovisuales de entrevistas a referentes del MNCP, la CTA y la CGT, todos videos institucionales de muy difícil acceso, dado que no se encuentran disponibles libremente en internet, sino en ciertos sindicatos, casas de los realizadores o archivos de algunas de las instituciones. Al mismo tiempo, citamos fragmentos del documental "Una modesta proposición” filmado por Miguel Matoํㅡㄹ quien acompañó al Movimiento en el año 2001 para registrar su trabajo y recomponer la historia de los chicos y chicas de la calle.

Nos interesa rescatar la posición de los sujetos en la historia (Bloch, 1982; Fontana, 1999), para lo cual las entrevistas con los protagonistas han funcionado como herramientas apropiadas, al igual que los videos. Sin embargo, estos relatos personales corren el riesgo de teñir el pasado de una impronta demasiado individual o demasiado direccionada a una intencionalidad político-coyuntural. Para evitar caer en ese error es que cruzamos estos relatos con bibliografía teórica y rescatamos en 
cada momento el hecho de que "las formas de organización que históricamente se constituyen no son simplemente resultantes de la acción individual de algunos dirigentes o grupos sindicales o políticos” (Klachko, 2009, p. 205).

En este mismo sentido, utilizamos también materiales periodísticos de la época, que aportan un registro claro de fechas que se encontraban mezcladas en las entrevistas realizadas y videos, así como también brindan ciertas estadísticas y colaboran con la construcción de criterios propios sobre los niveles de impacto de las acciones del Movimiento y los y las trabajadoras. Asimismo, citamos algunos fragmentos de la tesis de doctorado en elaboración de la colega Gabriela Scarfó (2016), quien, a partir de su investigación sobre infancia y trabajo, recopiló información de primera mano de organizaciones pertenecientes al MNCP, realizando un valioso aporte teórico a la reconstrucción histórica.

\section{3 - 1990. La infancia pobre como emergente social y las primeras articulaciones del campo popular}

Históricamente el campo popular ha desarrollado experiencias de trabajo con niños y niñas de la propia clase por fuera o en relación con los programas estatales. Por citar solo el caso argentino, podemos mencionar las bibliotecas populares de anarquistas y socialistas de principios de siglo XX, las organizaciones de pioneers comunistas en los años 30, las experiencias de apoyo escolar y comedores infantiles de todos los tiempos, los hogares, los espacios lúdicos, las propuestas productivas para generar recursos para los chicos y chicas de la calle, etc. (Camarero, 2007; Carli, 2012). La infancia pobre fue siempre una cuestión abordada desde las organizaciones sociales con diversos grados de articulación entre las múltiples experiencias territoriales y con la política pública, según el estado de la lucha de clases en cada contexto (Autora, 2017; Santillán, 2012). Por su parte, la burguesía gobernante también ha desarrollado a lo largo de la historia sus estrategias para lidiar con los niños y niñas “en peligro material o moral” (Ley de Patronato, 1919), centradas en la vigilancia e institucionalización de los y las menores, y que han sido analizadas en sus especificidades contextuales por diversas autoras (Cosse et al., 2011; Guemureman \& Daroqui, 2001; Villalta, 2005).

Particularmente, la primavera democrática invitó a muchos a acercarse otra vez a la vida política y enrolarse en actividades sociales, multiplicando estas experiencias locales de trabajo con niños y niñas (así como en otras propuestas de defensa de la democracia, los derechos humanos, etc.), en una década donde la infancia adquirió particular visibilidad tras haber sido botín de guerra de los genocidas en la última dictadura cívico militar (Carli, 2009). Sin embargo, el terrorismo de Estado fue también una derrota para la clase trabajadora, que fue amedrentada y diezmada, tanto en sus bases, como en parte de su dirigencia, y en sus expresiones más y menos combativas, retrocediendo en materia de legislación social y organización (Ghigliani, 2008). Asimismo, los y las trabajadoras debieron seguir luchando contra los ataques legislativos y de desprestigio de la gestión alfonsinista contra la organización obrera $y$, junto a los organismos de derechos humanos en auge, se enfrentaron a los innumerables avatares de los juicios a la Junta Militar, problemática central en los primeros años de radicalismo. 
Ni los sindicatos, ni las organizaciones de derechos humanos, ni ningún partido o movimiento popular pudo articular las luchas por "los pibes" (como los llaman muchas veces los referentes adultos) que, sin embargo, iban creciendo, porque crecía sin pausa la pobreza y miseria del pueblo. Por una fuerte presión de organismos de crédito internacionales, el gobierno "se vio obligado a realizar un ajuste ortodoxo atendiendo a las indicaciones del FMI. Estas medidas terminaron generando mayor inflación, de modo que los precios al consumidor acumularon un incremento del 700\% a lo largo de 1984” (Gaggero y De Luca, 2016). Este régimen republicano mostró rápidamente no poder cumplir con la consigna de que "con la democracia se come, se educa y se cura”, , y con el fin de la ilusión alfonsinista no solo entraron en jaque los partidos de gobierno, sino "los partidos con capacidad de organización y movilización de masas y los sindicatos reformistas y burocratizados (...) también [vieron] minada su propia capacidad de ejercer la dirección intelectual y moral” del campo popular (Campione, 2007, p. 137), y en ese marco “algo nuevo había que hacer, pero todavía no sabíamos muy bien qué era” (Silvina Vieyra, comunicación personal, 20 de abril de $\left.2016^{4}\right)$.

Florecieron entonces en forma aislada comedores, hogares y espacios de apoyo escolar como iniciativas locales de organizaciones populares, o en relación con iniciativas de trabajadores estatales involucrados en programas gubernamentales de infancia, que accionaban por fuera de los márgenes oficiales de su contrato. Estas experiencias han sido difíciles de registrar dada la pequeña escala a la que trabajaban y su poca sistematicidad, pero han quedado guardadas en las memorias de los protagonistas en frase como “y, era un momento así, la dictadura había dejado un país hecho pelota y la gente empezó a hacer comedores en sus casas. Daban comida, clases de apoyo, lo que hiciera falta” (Omar Giuliani, comunicación personal, 23 de noviembre de 2015); “a través de ATE [Asociación de Trabajadores del Estado] les dábamos galletitas, útiles, lo que había” (Víctor De Gennaro, comunicación personal, 15 de abril de 2016톨). Estas primeras organizaciones de infancia trabajaban con escasos recursos y una innumerable cantidad de problemáticas que atravesaban los niños, niñas y adolescentes de familias obreras, en el marco de un movimiento sindical en plena reorganización y sin una estrategia clara para abordar los nuevos desafíos de la democracia: "Pensábamos que la sociedad iba a volver al 76 pero no, era una sociedad nueva y había que plantearse nuevos problemas y soluciones” (Víctor De Gennaro, comunicación personal, 15 de abril de 2016).

En este contexto se destacan dos figuras pioneras, tanto en su forma de trabajar con los niños y niñas como en su insistencia por la unidad en la diversidad como estrategia de lucha privilegiada. El primero es Alberto Morlachetti, referente principal de la Fundación y Hogar Pelota de Trapo, que trabajó con chicos y chicas de la calle desde 1974. Allí se gestó el Movimiento Nacional Chicos del Pueblo (MNCP), que fue la primera experiencia de organización nacional con el objetivo de luchar por una infancia digna en términos clasistas, y de hacerlo con los niños y niñas como protagonistas de dicho proceso. En 1984, Carlos Cajade, cofundador del MNCP, comenzó a hacer el mismo trabajo en el Hogar Tres Veces Admirable de La Plata y se relacionó con Morlachetti en el Movimiento Renovador Peronista (MRP), una facción del Partido Justicialista (PJ), donde ambos militaban sin demasiada organicidad hasta entonces. Fue ese encuentro en el trabajo cotidiano de ambas organizaciones de infancia lo que consolidó su participación en el MRP y le otorgó a sus 
referentes cierto peso en la estructura del PJ.

Desde allí se ocuparon de tejer redes con otros espacios que trabajaban la niñez en relación con la necesidad de una transformación económica sustancial, para que las familias tengan trabajo y la riqueza se distribuya. Además, ambas organizaciones asumían la capacidad y la necesidad de que niños y niñas sean parte de esas luchas, de discutir con ellos su realidad y las posibles transformaciones, y de marchar con ellos cada vez que era necesario. En un contexto donde se había instalado la pregunta por la representación política y social en general, emergía la posibilidad de autorepresentación de los niños y niñas (Carli, 2009), y esto era portado por el Movimiento como una de sus principales banderas.

El MNCP también portó desde el comienzo la consigna por mejores condiciones de vida, en un país en el que los ajustes económicos implementados por la Junta Militar, y reforzados en democracia a través de acuerdos con el Fondo Monetario Internacional, estaban haciendo estragos en los bolsillos de los trabajadores, con un aumento sostenido del desempleo y subempleo (Bonnet, 2008). El PJ se autoproclamó en esa escena como una alternativa real ante el caos y triunfó así en varias elecciones, ubicando a Morlachetti como funcionario del Ministerio de Desarrollo Social durante la gobernación de Cafiero en la provincia de Buenos Aires (Telam, 2015), y luego en la intendencia de Grosso en Buenos Aires, de la que se alejó en 1990.

Podemos asumir, entonces, que estos fueron años en los que la pobreza pasó a ser un tema de la agenda pública, en una realidad económica que se desmoronaba, y allí la infancia pobre se legitimó como una problemática social en aumento, a tal punto que sus referentes fueron invitados a participar de la gestión estatal. Si bien es innegable que esta articulación debió poner en tensión los propios principios de participación infantil del Movimiento y su amplitud para tejer alianzas con organizaciones de niñez, Claudio Lozano $\underline{6}$ comentó al respecto "fue muy poco tiempo como para que haya quilombo, en seguida Alberto se fue del MRP, nos fuimos todos del PJ" (comunicación personal, 20 de mayo de 2016). Scarfó afirma en este mismo sentido que, incluso en esos años de gestión estatal de Morlachetti, el MNCP "se definía como independiente de cualquier organización política o religiosa. La única relación de pertenencia que los definía era con la clase trabajadora” (2016, s/p).

Con este escenario es que en 1988 el Movimiento Nacional Chicos del Pueblo, aún pequeño y concentrado en la Capital Federal y la Provincia de Buenos Aires, organizó un encuentro entre los niños y niñas de las diversas organizaciones que lo componían para que ellos expliquen e intercambien sus principales problemáticas y busquen soluciones junto a los referentes del Movimiento. Este fue el "Primer Congreso de los Pibes de la Calle”, síntesis de más de una década de lucha por la articulación entre las experiencias de trabajo con la infancia pobre desde una perspectiva clasista.

El evento consistió en dos encuentros, el primero tuvo lugar en la sede de la Asociación de Trabajadores del Estado y fue más reducido en número. El segundo se realizó en la sede de Brasil de la Confederación General del Trabajo (CGT), ya con el nombre de "Congreso Nacional de los Pibes de la Calle. Sin trabajo no hay infancia” (CGT, 1988). La elección del lugar no fue azarosa. Tal como lo indica el nombre del evento, para el Movimiento era cada vez más obvia la relación 
entre el modelo económico y las problemáticas de la infancia pobre, tanto que ya para ese entonces había adoptado como lema distintivo la famosa frase de Cajade: "atrás de cada pibe de la calle hay un padre desocupado”. El MNCP hizo así una primera gran apuesta por visibilizar la problemática de la infancia en relación con el trabajo, en el contexto de una CGT ya unificada tras los conflictos suscitados por las intervenciones dictatoriales (Fernández, 1996), y oficialmente peronista (aunque sin una hegemonía clara al interior), que en esos años "resultó capaz de centralizar unas luchas sociales que se desplazaban cada vez más de las demandas políticas a las económicas y sociales” (Bonnet, 2008, p. 181).

Fue específicamente un sector del movimiento obrero organizado en la CGT el que respondió a esta situación otorgándole "la casa de los trabajadores para los pibes” (Omar Giuliani, comunicación personal, 04 de abril de 2016), comenzando así a asumir esa lucha: “Se empieza a asociar el problema del trabajo a todas las cuestiones de los pibes (...) que era una realidad que no la habíamos dimensionado” (Víctor De Gennaro, comunicación personal, 15 de abril de 2016). Este grupo comenzaba a asumir las necesidades de una transformación en las formas de lucha sindical y disputaba la hegemonía dentro de la central obrera, de la mano de Víctor de Gennaro y Germán Abdala, ambos dirigentes del combativo y muy golpeado sector estatal, que quedará afuera de la nueva estructura del PJ con el triunfo menemista (Gaudio y Thompson, 1990). Es este sector político, acompañado de la figura de Claudio Lozano, militante del peronismo y de la SERPAJ (donde la preocupación por los niños y jóvenes pobres también comenzó a ser una prioridad), el que le da lugar a la cuestión de la infancia. Víctor de Gennaro recuerda al respecto:

Es Morlachetti con Claudio [Lozano], (...) un congreso de chicos que se hace en la CGT que es distinto, con el aval de Ubaldini, él está al final hablando, se le subían los pibes a la cabeza [se ríe] (...) No es que lo organizamos nosotros [los trabajadores], como lo va a hacer después, una nueva visión que va a tener la Central, la organización era de Morlachetti y nosotros le dábamos el local (...). El sindicato peleando por los pibes, eso era muy nuevo para nosotros (comunicación personal, 15 de abril de 2016).

El video del Congreso, realizado por la propia central obrera, finaliza con una placa en la que se lee “ellos son nuestros hijos” y el logo de la CGT. En el transcurso del video se ve al Titular del Departamento de DDHH de la CGT, Ricardo Pérez (del sindicato de camioneros), explicitar también la relación entre el trabajo y la infancia pobre:

Esta marginalidad social que significa los chicos de la calle, que tienen un tratamiento como delincuentes, significa crear un modelo de país donde un pequeño sector de la sociedad concentrara la riqueza que se produce y dejar al resto de la sociedad argentina absolutamente marginada (CGT, 1988).

El primer encuentro de los chicos fue organizado como un plenario con paneles, donde Claudio Lozano recuerda que "fue uno de los momentos más raros de hablar en público (...) y la verdad es que vos escuchabas hablar a los pibes de su relación con la calle, con las drogas y decías ¿qué les voy a decir yo?” (comunicación personal, 20 de mayo de 2016). En todas las entrevistas realizadas al respecto el relato es similar: "nos subordinamos a la lógica de los pibes (...) fue todo un aprendizaje estar con ellos” (Silvina Vieyra, comunicación personal, 20 de abril de 2016). Ya en este 
punto hay algo particular en la perspectiva de infancia (que reedita viejas experiencias de lucha en contextos contemporáneos), ubicando a niños y niñas en primer lugar, como protagonistas de su realidad. Esta intención puede verse claramente en el video del evento, donde se muestran a niños, niñas y jóvenes en reiteradas escenas conversando en pequeños grupos sobre sus terribles experiencias en los hogares y en las calles, donde enfrentaban una persecución policial permanente: "no sos libre de ir a la esquina a tomar una coca porque viene la policía y te agarra y ya está, perdiste a libertad” (CGT, 1988).

La crítica a la violencia institucional es permanente en los testimonios de los niños, niñas y jóvenes que deja ver este material audiovisual, donde se intercalan testimonios de chicos, chicas y grandes, donde todos los y las adultas eran referentes obreros del momento. Luego, el video exhibe algunas imágenes del plenario general de niños y niñas, dirigido por ellos mismos, donde se escucha a una de las pequeñas representantes de unos 15 años gritar al micrófono:

A la sociedad no le importan nuestros problemas porque saben que tienen a la noche una cama limpia donde dormir, sin importarle que nosotros vamos a dormir en la calle, sin importarle que somos golpeados y torturados por la policía, porque ellos no lo entienden, porque tienen dinero y se creen más gente que nosotros, pero nosotros somos de la calle y valemos más (CGT, 1988).

A partir de este congreso es que algunos trabajadores y trabajadoras comenzaron a pensar en la posibilidad de plantear, junto a sus reivindicaciones obreras, "una política hacia la infancia asociada a un cuestionamiento del modelo vigente, de la infantilización de la pobreza” (Claudio Lozano, comunicación personal, 20 de mayo de 2016), un discurso que también se escuchaba en el paro docente masivo del mismo año, al que habían asistido algunas de estas organizaciones de niñez a dar su apoyo, asumiendo que todas las luchas abonan a la transformación social necesaria para el bienestar de los chicos “en sus casas, sus escuelas, las plazas”, etc., según el relato de Víctor sobre aquellos años (comunicación personal, 15 de abril de 2016). Estos cuestionamientos al sistema, y la negativa de los gobernadores y funcionarios de hacer algo al respecto, exacerbaron la crisis de participación, que estos dirigentes ya habían gestado, sobre un proyecto cada vez más cercano al neoliberlaismo, como se encontraba el PJ en ese momento ${ }^{7}$. El partido termina de expulsar de su seno a los referentes sociales en tan solo unos meses: "nosotros en el indulto [a los militares] en el ochenta y nueve, noventa, renunciamos al PJ. Víctor, Germán $\underline{8}$, Alberto, yo, nos fuimos todos” (Claudio Lozano, comunicación personal, 20 de mayo de 2016). De modo que todos se retiraron del partido de gobierno y del gobierno del Estado iniciando una nueva etapa en la lucha sindical y social.

Este Congreso, en tanto acto de solidaridad de los y las trabajadoras hacia las organizaciones de infancia, fue el primero de muchos que llevó finalmente a un trabajo realmente colectivo, ya en la siguiente década y por fuera de la CGT. Se cierra así un primer período de gestación de las organizaciones de infancia, que surgieron como iniciativas locales que daban cuenta de una problemática que iba en aumento y un sector de la clase dispuesta a luchar por contrarrestar sus efectos, pero con pocas herramientas para hacerlo. El encuentro de estas experiencias en el Movimiento Nacional Chicos del Pueblo fue, entonces, un salto cualitativo en organización y 
desarrollo, obtención de recursos y de una legitimidad de la causa en ascenso, producto también del aumento en la pauperización de la vida de las familias obreras. A su vez, el sindicalismo se encontraba debilitado tras haber sufrido el terrorismo de Estado y en una encrucijada de internas, que no le permitía avanzar demasiado, a pesar de haber logrado articular diversas luchas y obtener variadas victorias.

El encuentro entre estos dos colectivos fortaleció a ambas partes, aunque en términos más modestos para el sindicalismo. El MNCP hizo una gran puesta en escena con una actividad masiva y una campaña de propaganda, como nunca antes había tenido: "para el movimiento fue espectacular, imagínate, había cámaras, estaba Ubaldini” (Silvina Vieyra, comunicación personal, 20 de abril de 2016). Omar explicaba: “ya para ese momento ellos estaban afuera del partido [justicialista], eran funcionarios, pero de arriba les cortaban las piernas, no podían hacer nada. De ahí en seguida se fueron” (Omar Giuliani, comunicación personal, 23 de noviembre de 2015). Este grupo de la CGT les brindó entonces los recursos y la visibilidad que no tenían, a la vez que buscaban instalarse ellos mismos como un sector del movimiento obrero "más preocupado por la cuestión social” (Claudio Lozano, comunicación personal, 20 de mayo de 2016) y con la fuerte intención de "incluir a otros grupos, los desocupados (...) los problemas de los chicos” (Ricardo Perez en el video de la CGT, 1988).

\section{0-2001. La infancia pobre como problemática de clase y la construcción de una lucha unificada}

El año 1990 es, para el Movimiento, de consolidación como organización popular en oposición a (y con una fuerte denuncia contra) los partidos y los gobiernos con los que había trabajado hasta entonces, cada vez más convencido de que "el capitalismo no tiene rostro humano”, otra de sus frases más populares. A partir de este momento, el MNCP atravesó un proceso de radicalización en sus discursos y prácticas por una sociedad de iguales, de la mano de diversidad de sectores en lucha contra las estructuras de poder y sus efectos sobre la clase trabajadora en general y los niños y niñas en particular.

La nueva hegemonía menemista, dentro del PJ y como proyecto político nacional, representó el inicio de un proceso de avance del capital sobre el trabajo sin precedentes en la historia nacional. La clase dominante forjó un nuevo tipo de Estado basado en la convertibilidad como estrategia de acumulación y un proyecto hegemónico que respondía a los intereses de la gran burguesía aperturista y financiera (Bonnet, 2008). La implementación de este programa significó un aumento de la tasa de explotación a partir de una pérdida de derechos laborales, una baja en los niveles de empleo formal y una descentralización de las funciones y recursos estatales. Esto redundó en la concentración de la riqueza en pocas manos y la consecuente pauperización de la vida de la clase obrera en general y de la infancia proletaria en particular, en tanto se produjo "una creciente desprotección de las familias, que erosionó sus capacidades para brindar condiciones básicas de desarrollo a niñas, niños y adolescentes” (CEPAL y UNICEF, 2005, p. 27).

Estos mismos organismos internacionales, basados en los datos de la Encuesta Permanente de Hogares (INDEC) y de la Dirección Nacional de Cuentas Nacionales, publicaron en 2006 que la pobreza infantil creció del 20\% al 39\% en términos absolutos entre 1992 y 1999, y a una velocidad 
mayor que la pobreza total del país. En el informe de 2005, las mismas agencias publicaron que entre 1990 y 2002 hubo un aumento del 10, 5\% de la pobreza relativa infantil (en relación a los consumos de la propia sociedad y no de la cobertura de necesidades básicas), siendo uno de los países record en la región. En esos mismos años, la desigualdad general aumentó en forma sostenida, en tanto "se incrementó la porción del ingreso nacional percibida por el 10\% más rico de la población” (CEPAL y UNICEF, 2006, p. 8) y disminuyó el ingreso del 10\% más pobre. Además, el desempleo llegó casi al 20\% para fines de la década del 90 y la población bajo la línea de pobreza alcanzó casi al 40\% (Elisalde, 2016).

En este contexto los jefes y jefas de hogar cubrían cada vez menos las necesidades básicas de cuidado de los más pequeños (salud, alimentación, abrigo, etc.), situación que redundó no solo en la pauperización de la vida de los niños y niñas de familias trabajadoras, sino también en la explosión del fenómeno de chicos y chicas en situación de calle (Carli, 2009) e insertos en el mercado laboral en las peores condiciones (Iñigo Carrera, 2008), además de un aumento significativo de la mortalidad infantil (Iñigo Carrera et al., 1995). Otra de las grandes problemáticas generacionales de la década, y que fue fuertemente denunciada por las organizaciones sociales, resultó el encierro de estas infancias como política de Estado frente a la infantilización de la pobreza: "va dando forma a la industria de la institucionalización (...) se produce un aumento del ingreso de niños y adolescentes en instituciones de minoridad por causas sociales” (Carli, 2009, p. 27). En el nuevo Estado que abandonaba las recetas del bienestar, esta violencia se renovó como forma de relación para con los y las menores de sectores populares, que fueron catalogados como peligrosos, y por ello perseguidos y maltratados por la policía y, cada vez más, asesinados por ella (Verdú, 2009).

En este contexto se inicia también un período nuevo para las organizaciones obreras. Las formas sindicales de lucha, protagonistas de la década del 80 y principios de los 90, comenzaron a convivir con nuevos sujetos participando de la lucha de clases con novedosas demandas y modos de organización y lucha, que surgieron de aquellas más tradicionales (Campione, 2007). Entonces, las formas sindicales se articularon con otras no-sindicales, como las movilizaciones de pueblos enteros contra los cierres de puestos de trabajo en fábricas y minas, la ocupación de tierras y las marchas contra el gatillo fácil, el reclamo de subsidios, la mejora de pensiones jubilatorias, etc.: “de los piquetes en la puerta de las empresas a los cortes de la calle y las rutas, como nuevos modelos de lucha” (Bonnet, 2008, p. 350).

Esto significa que las nuevas condiciones de precarización modificaron también a los espacios de lucha, con un corrimiento de la fábrica como lugar privilegiado de conflicto hacia los pueblos y barrios, otorgándole un carácter territorial a las disputas, que contribuyó a pensar la infancia como problemática de clase, pero también como sujeto político. Y es esa infancia ya nucleada en el MNCP la que comienza a desarrollar un nuevo rol en la pelea por su supervivencia en las calles, en las plazas y en los hogares, haciendo suyas las luchas y modos de lucha de la etapa. Ya desde comienzos de los años 90 organizaron marchas pidiendo presupuesto para los comedores y hogares, a la vez que el aumento de sueldo para los trabajadores estatales (llamados "educadores" o “referentes”), que se hacían cargo de esos lugares y de los chicos y chicas que los habitaban. Así los chicos y chicas salieron a ocupar el espacio público y a reclamar con su propia voz por una vida mejor de la mano de los sindicatos. 
También debemos considerar el hecho de que las propias familias obreras comenzaron a salir a la calle con todos sus miembros a luchar por trabajo y, cada vez más, por comida, generando una fuerte irrupción de mujeres y niños y niñas en los espacios de disputa, muchos porque querían acompañar a sus padres y madres, y otros porque estaban a cargo de aquellas mujeres combativas, que resignificaron sus tareas de cuidado en el nuevo contexto de adversidad $\stackrel{9}{\text {. }}$

Estas articulaciones no fueron una simple sumatoria de reclamos, sino que el encuentro modificó las características de uno y otro grupo, transformando la relación que significa la lucha de clases. Por su parte, el sector del movimiento obrero organizado, que había roto lazos con el PJ, también lo hizo en 1992 con la CGT, forjando un modelo sindical alternativo consumado en la Central de los Trabajadores de la Argentina en 1992, a partir de la cual los sectores obreros más combativos "intentaron articular esas luchas sociales, recuperando de manos de la CGT oficial una función a la que ésta ya había renunciado” (Bonnet, 2008, p. 351) y por ello habilitaron la afiliación directa y así la inclusión de organizaciones sociales y de grupos específicos, como es el caso de los y las jubiladas y los y las niñas (Elisalde, 2016). Así, problemáticas surgidas en el período fueron asumidas creativamente por la clase trabajadora, y combatidas con nuevas perspectivas y estrategias.

El referente principal del MNCP, Alberto Morlachetti, fue desde el primer momento el Secretario Nacional de Derechos Humanos de la Central, y desde allí impulsó diversas campañas contra el maltrato infantil en instituciones y la violencia policial hacia chicos y chicas en las calles, así como también se ocupó de denunciar la falta de trabajo de los adultos como principal problema de los niños: "que se vea la problemática de los pibes era que se vea la problemática del desempleo, que se vea la Central en la calle” (Claudio Lozano, comunicación personal, 20 de mayo de 2016). La Central, que crecía como proyecto político en ese entonces, fue otra vez para el Movimiento una articuladora que le brindó contactos de otras organizaciones en todo el país, principalmente a través de los sindicatos estatales (dirigidos por De Gennaro y Abdala) y docentes (con Yasky a la cabeza), que representaban las bases más sólidas de este nuevo modelo sindical y le garantizaban al proyecto un piso nacional: "vas a cualquier rincón del país, ¿y qué tenés? Un maestro y un trabajador del Estado seguro. Bueno, con ellos íbamos a construir también el Movimiento” (Omar Giuliani, comunicación personal, 23 de noviembre de 2015).

De Gennaro explica que, en un primer momento, hubo entre ambas organizaciones "una cuestión de solidaridad más que de cuestión consciente organizativa”, y cuenta cómo "íbamos a frenar las represiones contra los pibes” (comunicación personal, 15 de abril de 2016), mientras un entonces niño del MNCP recuerda cómo los chicos y chicas acompañaban las marchas de los y las trabajadoras, y hasta las resistencias de los desalojos de los locales sindicales que el menemismo pretendía arrebatar:

Me acuerdo cuando fuimos a bancar el desalojo del local (...), se querían quedar con el edificio (...) éramos un montón de pibitos (...) veías pasar los billetes y te decían te lo compro, te lo compro, y era mucha plata, pero sabíamos que era todo mentira, que solamente nos querían sacar de ahí, pero no pudieron, no los dejamos (Ernesto, comunicación personal, 18 de abril de 2016). $\underline{10}$ 
Los relatos de los protagonistas dan cuenta de un encuentro cada vez más cotidiano entre sindicatos y Movimiento, y de un trabajo con los niños y niñas en pos de generar un diálogo intergeneracional donde los y las más pequeñas pudieran, no solamente decir lo que pensaban, sino comprender las razones por las cuales acompañaban un piquete o una toma de un edificio: "sabíamos que había que bancarlos porque ellos nos bancaban a nosotros, venían a nuestras marchas, éramos compañeros” (Ernesto, comunicación personal, 18 de abril de 2016). Más allá de lo que sucedió después con la Central y con el propio Movimiento, consideramos fundamental esa articulación en el proceso de transformar a niños y niñas en protagonistas de las luchas, en “compañeritos”, asumiéndolos como sujetos políticos sin perder de vista las necesidades particulares de esa etapa de la vida: "marchaban, pero iban a la escuela todos los días" (Víctor de Gennaro, comunicación personal, 15 de abril de 2016). Morlachetti insistía en este punto, transformando las instancias de lucha en fiestas populares y sosteniendo la ternura en medio del conflicto: "Porque hubo caricias. Porque intentamos los insumos básicos de la crianza humana: la ternura, el pan, escuela, dignidad, belleza, invocar al ratón de las monedas, vestirse de guardapolvo blanco y danzar cuando los reyes anuncian regalos” (Morclachetti, en CTA, 2008).

Entendemos que en estos nuevos discursos y prácticas se gestó una novedosa categoría de infancia, una noción ensanchada de lo que puede ser esta etapa de la vida, que sin corresponder a los estereotipos normalizados, brindó a chicos y chicas la dignidad que en otras instancias la sociedad les estaba arrebatando. Este sector del movimiento obrero organizado comenzó a plantear entonces un modelo de infancia que permitía elaborar una imagen de niñez propia del campo popular, articulada con el resto de las luchas, ampliando así el propio concepto de clase:

Ese reconocimiento del cambio de ser chicos de la calle a hijos del pueblo. Que para mí es un cambio muy sustancial, entender que la calle no es capaz de parir hijos y que no hay hijos de la calle, sino que son hijos de nuestro pueblo (Víctor de Gennaro, comunicación personal, 15 de abril de 2016).

Podemos decir, entonces, que el MNCP se fue transformando para asumir como propias las luchas de todos estos trabajadores y trabajadoras organizadas, mientras ellos y ellas iban incorporando los reclamos de los y las más chicas a su identidad obrera. Así es que la Central reafirmaba en el mismo proceso su propuesta de modelo sindical alternativo y amplio de construcción social, y hasta forjaba una estética propia de la mano del Movimiento: "nuestras barricadas son de globos y títeres, es el amor y la ternura” (Morlachetti, en Modesta proposición, 2001). Y tanto era así que puede verse en las fotos de las marchas cientos de chicos y chicas caminando con sus coloridos globos, narices de payasos y tambores que repiquetean alguna canción como "Acaso porque soy niño tu no me vas a escuchar (...) Los niños desde La Quiaca marchan a la Capital, lo hacen para que sus derechos se puedan escuchar" (Modesta Proposición, 2001). En este punto queremos detenernos en la complejidad que significó este cambio para un sector de la clase obrera organizada, que debió refundar su identidad de la mano de desocupados, jubilados y niñeces, interpelando el propio concepto de clase para incluir a estos grupos (Campione, 2007) y tensando los posibles alcances de una central sindical que pretendía ahora defender los más variados intereses del pueblo, desde las meretrices organizadas $\underline{\underline{11}}$ hasta los ambientalistas en contra de las minas, sin desconocer las problemáticas propias de los trabajadores formales. 
En 1997 el Movimiento realizó la primera actividad de carácter nacional, una serie de "bicicleteadas" en diversas ciudades donde los chicos y chicas fueron protagonistas, no solamente porque manejaron sus bicicletas al frente de la movilización, sino también porque pintaron sus carteles y dieron las entrevistas a la prensa, en sintonía con las nuevas formas de lucha y demandas populares que mencionamos anteriormente. Con esta misma impronta se organizó en el año 2000 la Marcha Grande por el Trabajo desde Rosario a Buenos Aires, “donde los chicos ya si son protagonistas de las luchas de la clase obrera, ahí si ya no hay diferencia, nos incorporamos mutuamente” (Claudio Lozano, comunicación personal, 20 de mayo de 2016).

Esta marcha contribuyó a la legitimidad de los y las chicas en la calle, luchando con los y las adultas hacia el interior de la propia Central porque, tal como relata uno de sus referentes, “costaba muchísimo visibilizar al chico como un compañero, más bien se lo veía como algo lindo, como si fuera un complemento de la marcha", pero en la marcha "se ganaron el derecho de encabezar el 9 de agosto del año 2000 la Plaza de los Dos Congresos” (educador del MNCP, en Del Frade, 2010, p. 68). En los discursos centrales, se escucha a los referentes sindicales mientras se ve a los muñecos del Movimiento (distintivo de todas sus actividades), también se muestra a los chicos y chicas subidas al escenario central y se hace mención a ellos en más de una ocasión (CTA, 2000), reafirmando la identidad común que poseían ya a esa altura. Finalmente, en 2001, organizaron la Primera Marcha Nacional del MNCP, que partió desde la Quiaca y llegó a Plaza de Mayo con 25 mil personas reclamando por una infancia digna (Bruschtein, 2005), con la presencia de las Madres de Playa de Mayo y cientos de organizaciones sociales y de trabajadores organizados.

El estado de la lucha de clases en el campo particular de la infancia, tal como lo analizamos aquí, no fue solamente construido por los sectores más combativos, sino que debe ubicarse en el contexto de una fuerte reforma legislativa en lo que a niños y niñas respecta. En el año 1989 se firmó la Convención por los Derechos del Niño (CDN); este nuevo acuerdo internacional, firmado por todos los países del mundo salvo EEUU y Sudán del Sur, propuso el enfoque de derechos como nuevo abordaje de las problemáticas de infancia, ubicando a niños y niñas como ciudadanas y al Estado como garante de sus derechos. En 1990 el pacto se ratificó en Argentina, convirtiéndose en la ley nacional $\mathrm{N}^{\circ} 23.849$ con el apoyo de casi todo el arco político local, la iglesia y UNICEF. La CDN descartó el enfoque explícitamente represivo hacia la minoridad que se desarrolló históricamente en el país y en el mundo, proponiendo un abordaje que abrió espacios de autonomía para la infancia y que, en el marco de la lucha de clases, fue una herramienta más de las organizaciones sociales.

Este documento colocó a nivel global y oficialmente a los niños y niñas como sujetos de derecho: "recién sobre el final del siglo XX los niños fueron reconocidos en su subjetividad jurídica y política, como últimos actores sociales invitados a sentarse a la mesa de la ciudadanía” (Beloff, 2008, p. 70). A partir de estas modificaciones los niños y niñas ya no debían ser considerados inferiores a los y las adultas, como hasta entonces, sino sujetos plenos con sus propios derechos y con la capacidad de reclamarle al Estado por la garantía de los mismos. Además, la Convención exhortó desde el primer momento a los Estados a hacerse cargo de la población infantil a través de una política pública integral y no exclusivamente desde la justicia, que castigaba con el encierro a los niños pobres. Este nuevo abordaje también valoró la participación infantil, que se menciona en diversas ocasiones en el texto de la CDN: “fomentando la participación activa de las niñas, niños y 
adolescentes” (artículo 9); “faciliten la participación activa del niño en la comunidad” (artículo 23); “el derecho del niño a participar plenamente” (artículo 31); etc. También se menciona aquí el derecho a opinar y ser oídos por los adultos cuando se trata de decisiones que hacen a sus propias vidas: “expresar su opinión libremente en todos los asuntos que afectan al niño” (artículo 12).

Las críticas y limitaciones de la CDN abundan y refieren tanto a sus concepciones más teóricas (Barna, 2013) como a su implementación en la Argentina (Beloff, 2008). En definitiva, es imposible que una legislación burguesa, que se sostiene sobre un Estado capitalista existente en tanto forma de relaciones de dominación de clase, logre garantizarle una vida digna a los niños y niñas de clase trabajadora, dado que eso requeriría el bienestar de toda la familia obrera y, así, el fin de la propia sociedad de clases que le da origen a la ciencia del derecho: "cuyo contenido está dado siempre por las relaciones de esta clase, como con la mayor claridad lo demuestran el derecho privado y el derecho penal” (Marx, 2014, p. 386). A pesar del carácter reformista de cualquier cambio legislativo, rescatamos la CDN como un avance en materia de infancia que eliminó viejas y habilitó nuevas prácticas y discursos sobre y con niños y niñas, que se pusieron en juego colaborando con su posicionamiento como sujetos políticos.

En el marco de una fuerte derrota del campo popular nos encontramos, sin embargo, con un avance en la organización y lucha popular que hizo crecer en su propia dinámica la lucha por la infancia digna. En una articulación sin precedentes entre el movimiento obrero y la infancia pobre, se fortaleció la legitimidad de la causa y el nivel de organización alrededor de ella, lo que a su vez hizo crecer la nueva estrategia obrera de la CTA, acompañando sus marchas y brindado sus cuadros a la Central. Este proceso garantizó, en sí mismo y en el marco de la progresista CDN, uno de los derechos más vulnerados de la infancia pobre, que es el de participar en las decisiones que hacen a su propia vida, la posibilidad de opinar y de decidir, casi siempre coartada por la doble subsunción de ser niños y pobres.

\section{Conclusiones}

El recorrido presentado en este trabajo atraviesa dos momentos históricos de la historia argentina, caracterizados por el conflicto y la pauperización de la vida de la clase obrera, cada uno con sus particularidades políticas y económicas. En estos contextos, las luchas de las centrales sindicales y de las diversas organizaciones sociales adquirieron dinámicas particulares, de las que solo analizamos aquí una arista, que es aquella en la que se encuentran estos dos colectivos con la problemática específica de la infancia y las formas que adquieren en la articulación por la niñez digna.

En un primer momento (1983-1990) las interacciones entre el sindicalismo y el MNCP son aisladas y específicas de ciertas necesidades, mas no de un proyecto conjunto de trabajo y de lucha. Tanto el movimiento obrero como las organizaciones transitaban un período de entusiasmo por la vuelta de la democracia, pero también de fragmentación y reorganización (con algunos referentes y facciones que terminan incluso en las filas de las patronales), que a su vez se complejizaba por las condiciones de empobrecimiento que imponía el capital local en complicidad con el internacional. Sin embargo, el trabajo en conjunto que se realizó para el "Primer congreso de los pibes de la calle" le dio visibilidad a la problemática y cierta fortaleza a la propuesta de una nueva central, que pueda 
articular reclamos de diversos sectores de la clase. El segundo momento (1990-2001) estuvo marcado por la concentración de la riqueza en pocas manos y la consolidación de la hegemonía menemista como proyecto neoconservador, que transformó las configuraciones del mundo del trabajo. Además de la pauperización, estos procesos produjeron nuevas relaciones en el marco de la lucha de clases, profundizando las articulaciones en el propio proceso de disputa, fortificando cada reclamo particular, pero conformando, a su vez, una misma lucha, que se sintetiza en la frase: “Atrás de cada pibe de la calle hay un padre desocupado". Fue un período de crecimiento para ambos colectivos y de mucha actividad, en las calles y hasta en los medios de comunicación, lo que da cuenta de cierta legitimidad en aumento.

Creemos importante aclarar, aunque resulte obvio, que la historia del Movimiento y su relación con la Central no terminan en 2001, sino que, muy por el contrario, se continúa en una etapa de nuevas marchas nacionales (en 2002, 2005 y 2007) y nuevos avances legislativos (como la ley 26.061 de 2006), en un escenario de pobreza y conflictividad social. Para 2004 el porcentaje de niños pobres era casi del $40 \%$ de la infancia total (Lozano et al., 2005) y el Movimiento reunía a unos 35 mil niños de todo el país de unas 400 organizaciones, de los cuales 500 acompañaron todo el proceso de marcha al año siguiente (Bruschtein, 2005). Después del 2007 la situación de la infancia se modificó, al igual que la situación de la lucha de clases, y allí se reconfiguró este escenario, que esperamos poder analizar en detalle en un futuro trabajo.

Podemos afirmar, a modo de cierre, que en el avatar de las luchas y las coyunturas que se sucedieron entre 1983 y 2001, se desarrollaron diversos procesos complejos y entramados, de los que podemos extraer una interpelación al concepto de clase de las lecturas más tradicionales, donde se reconsideraron ciertos reclamos específicos de mujeres, niños y niñas y jubilados, ensanchando el propio concepto y aportándole una diversidad enriquecedora. Al mismo tiempo, y en absoluta relación, se vio interpelada la noción de infancia, que comenzó a dar lugar a estas otras formas de ser niño, atravesadas por crianzas colectivas y combativas en organizaciones sociales, sobre las que invitamos a seguir reflexionando para construir análisis, e incluso política pública, que contenga esta pluralidad.

\section{Notas}

1 Omar Giuliani es referente de una organización de infancia, que fue parte del MNCP y hoy continúa en el mismo camino. Sus entrevistas fueron realizadas el 23-11-2015 (en la organización de niñez donde trabaja) y el 04-04-2016 (en la CTA). Agradecemos su tiempo y disposición para la realización de este trabajo.

$\underline{2}$ Agradecemos especialmente al director por compartir este material para llevar adelante la investigación.

$\underline{3}$ Discurso de asunción del Presidente Raúl Alfonsín, 1983. Citado en: http://www.educ.ar/sitios/educar/recursos/ver?id=129085, visitada 23-03-2016

4 Silvina Vieyra es militante del sindicato de estatales (ATE) desde comienzos de los años 80 y 
desde allí acompañó el proceso de fundación de la CTA. Participó del primer Congreso de los Chicos de la Calle en 1988 y luego en todas las actividades del MNCP. Su entrevista fue realizada el 20-04-2016 en el sindicato. Agradecemos su tiempo y disposición para la realización de este trabajo.

$\underline{5}$ Víctor de Gennaro es referente sindical de la Asociación de Trabajadores del Estado desde los años 70s. Como oposición a la dictadura y a las subsiguientes presidencias, en 1992 rompió con las dirigencias de la CGT y fundó, junto a Germán Abdala, una central obrera alternativa, la CTA. Desde entonces condujo ese espacio, participando activamente de la vida del MNCP. Su entrevista fue realizada el 15-04-2016 en su espacio de militancia. Agradecemos su tiempo y disposición para la realización de este trabajo.

6 Claudio Lozano fue referente del SERPAJ desde fines de los 70s y trabajó desde entonces con ATE. Desde su conducción, también fue parte de la fundación de la CTA, donde ocupó diversos cargos de conducción, llegando a ser Diputado Nacional en un frente apoyado por este sector. Su entrevista fue realizada el 20-05-2016 en su espacio de militancia. Agradecemos su tiempo y disposición para la realización de este trabajo.

Z Son varios los autores que se ocupan de describir las disputas hegemónicas dentro del peronismo en las que gana una mirada desindicalizada y neoconservadora del partido (Pucciarelli, 2011; Schulz, 2014).

$\underline{8}$ Germán Abdala rompe el bloque peronista de diputados, del que era parte, y conforma lo que se constituyó como el Grupo de los Ocho, en franca oposición al oficialismo del PJ.

9 Si bien creemos que habría que profundizar el análisis del rol femenino en estos procesos, es innegable la relación que ello tiene con el lugar de la niñez en el conflicto, así como sucedió en la Huelga de las Escobas y varias otras manifestaciones obreras de principio de siglo XX, donde también mujeres y niños/as fueron protagonistas (Barrancos, 2008; Carli, 2012).

10 Ernesto fue parte del MNCP de pequeño y hoy es referente/educador de una organización de infancia, que continúa el mismo camino. Su entrevista fue realizada el 18-04-2016 en la organización

11 La Asociación de Mujeres Meretrices de la Argentina (AMMAR) se fundó en el año 1994 y en 1995 se sumó a la CTA con el objetivo de defender los derechos de las trabajadoras sexuales desde una perspectiva regulacionista, algo que no debatiremos en este artículo.

\section{Bibliografía}

Asamblea General de las Naciones Unidas (1989). Convención sobre los Derechos del Niño. http://www.un.org/es/events/childrenday/pdf/derechos.pdf, visitada el 22-04-2016

Barna, A. (2013). Del peligro moral y material al sujeto de derecho universal. La gestión de la niñez en perspectiva (Tesis de maestría en Diseño y Gestión de Políticas y Programas Sociales), FLACSO 
Barrancos, D. (2008). Mujeres, entre la casa y la plaza, Buenos Aires: Sudamericana,

Beloff, M. (2008). Reforma legal y derechos económicos y sociales de los niños: las paradojas de la ciudadanía. En Sarmento, D., Ikawa, D., Piovesan, F. (coords.), Igualdade, diferença e direitos humanos. San Pablo: Lumen Juris.

Bloch, M. (1982.) Introducción a la historia. Buenos Aires: Fondo de cultura económica.

Bonnet, A. (2008). La hegemonía menemista. El neoconservadurismo en Argentina, 1989-2001. Buenos Aires: Prometeo.

Bruschtein, L. (25-10-2005). La última pulseada del Curita. Página 12. Recuperado de: http://www.pagina12.com.ar/diario/contratapa/13-58381-2005-10-25.html

Camarero, H. y Herrera, C. (2005). El Partido Socialista en Argentina: nudos históricos y perspectivas historiográficas. En H. Camarero y C. M. Herrera (eds.), El Partido Socialista en Argentina. Sociedad, política e ideas a través de un siglo. Buenos Aires: Prometeo Libros.

Camarero, H. (2007). A la conquista de la clase obrera. Los comunistas y el mundo del trabajo en la Argentina, 1920-1935. Buenos Aires: Siglo XXI.

Campione, D. (2007). Para leer a Gramsci. Buenos Aires: Centro Cultural de la Cooperación.

Carli, S. (coord.). (2009). La cuestión de la infancia. Entre la escuela, la calle y el shopping. Buenos Aires: Paidós.

Carli, S. (2012). Niñez, pedagogía y política. Transformaciones de los discursos acerca de la infancia en la historia de la educación argentina entre 1880 y 1955. Buenos Aires: Miño y Dávila.

Central de Trabajadores de la Argentina (18-11-2013). Memorial del pueblo. Homenaje a Cajade y exposición de Morlachetti. Agencia CTA. Recuperado de: http://www.agenciacta.org/spip.php? page=imprimir articulo\&id article $=10537$

Central de Trabajadores de la Argentina (Productor y director). (2000). La marcha grande [película]. Argentina, Buenos Aires, https://www.youtube.com/watch?v=cBRl-ZB6Kek, visitado 30-04-2016

Central de Trabajadores de la Argentina (09-05-2008). Con ternura venceremos. Agencia CTA. Recuperado de: http://archivo.cta.org.ar/Con-Ternura-Venceremos.html

CEPAL y UNICEF (2005). La pobreza infantil en América Latina. Desafíos 1, Naciones Unidas.

CEPAL y UNICEF (2006). Efectos de la crisis en Argentina. Las políticas del Estado y sus consecuencias para la infancia. Documentos de difusión. CEPAL y UNNICEF.

Ciafardo, E. (1992). Los niños en la ciudad de Buenos Aires (1890-1910). Buenos Aires: Centro Editor de América Latina.

Confederación General del Trabajo (Productor y director). Primer Congreso de Pibes de la Calle [Película]. Argentina, Buenos Aires.

Cosse, I. (2006). Estigmas de nacimiento: peronismo y orden familiar, 1946-1955. Buenos Aires: Universidad San Andrés. 
Cosse, I., Llobet, V., Villalta, C. y Zapiola, M. C. (2011). Infancias: políticas y saberes en Argentina y Brasil. Siglos XIX y XX. Buenos Aires: Teseo.

Cucuzza, H. (1996). De continuidades y rupturas: el problema de las periodizaciones. Anuario de Historia de la Educación, 1, 1996/1997. San Juan: Fundación Universidad de San Juan.

Del Frade, C. (2010). La marcha grande. A diez años del rio místico de la historia argentina. Buenos Aires: CTA ediciones. http://ctanacional.org/dev/wp-content/uploads/2015/01/la-marchagrande.pdf, visitado el 13-04-2016

Elisalde, R. (2016). La era menemista y el gobierno de la Alianza: neoliberalismo, conflicto social y crisis (1989-2003). En Elisalde, García y Scher (comp.), Historia social de la Argentina Contemporánea 1930-2003. Buenos Aires: EUDEBA.

Fals Borda, O. (1990). El problema de cómo investigar la realidad para transformarla por la praxis. Bogotá: T.M Editores.

Fernández, A. (1996). Flexibilización Laboral y Sindicatos. Un Estudio de Caso. Estudios Sociales, 10(1), 209-219

Fontana, J. (1999). La historia después del fin de la historia, Barcelona: Critica

Gaggero, H. y De Luca, M. (2016). Argentina 1983-1989: Crisis y colapso del Estado. En Elisalde, García y Scher (comp.) Historia social de la Argentina Contemporánea 1930-2003. Buenos Aires: EUDEBA

Gaudio, R. y Thompson, A. (1990). Sindicalismo peronista, gobierno radical: los años de Alfonsín. Buenos Aires: Fundación F. Ebert.

Gentile, M. F. (2011). Niños, ciudadanos y compañeritos: un recorrido por los distintos criterios para el trabajo de inclusión social de niños y adolescentes de sectores vulnerables. En Cosse, Llobet, Villalta y Zapiola (comps), Infancias: políticas y saberes en Argentina y Brasil (siglos XIX y $X X)$. Buenos Aires: Teseo

Ghigliani, P. (2008). La noción de derrota en la historia reciente del movimiento obrero argentino. $V$ Jornadas de Sociología de la UNLP. Universidad Nacional de La Plata. Facultad de Humanidades y Ciencias de la Educación. Departamento de Sociología, La Plata.

Guemureman, S. y Daroqui, A. (2001). La niñez ajusticiada. Buenos Aires: Ediciones del Puerto.

Iñigo Carrera, J. (2008). Trabajo infantil y capital. Buenos Aires: Imago Mundi .

Iñigo Carrera, N., Cotarelo. M. A., Gómez, E. y Kindgard, F. (1995). La revuelta argentina 1989 1990. Documento de Trabajo $N^{\circ}$ 4, PIMSA.

Klachko, P. (2009). Transformaciones en la organización popular a partir de la participación en el gobierno del Estado. El caso del movimiento Barrios de Pie 2001-2008. En Documentos y publicaciones PIMSA 2009 (p. 202-234). 
Ley de Patronato de la Infancia (1919), Congreso Nacional de la Argentina http://23118.psi.uba.ar/academica/carrerasdegrado/psicologia/informacion adicional/practicas de i nvestigacion/775/legislacion/Ley\%2010903 patronato.pdf, visitada el 05-04-2016

Liebel, M. (2006). Entre protección y emancipación: derechos de la infancia y políticas sociales. Madrid: Universidad Complutense.

Lozano, C. (2002). Argentina: una comunidad en riesgo. Infancia, adolescencia y juventud en mayo del 2002. Buenos Aires: Instituto de estudios y formación de la CTA.

Lozano, C, Rameri, A y Raffo, T. (2005). El hambre es un crimen. Infantilización de la pobreza en la Argentina, Buenos Aires: Instituto de estudios y formación CTA

Marx, Karl. (2014). La ideología alemana. Madrid: Akal

Mato, M. (Productor y Director). (2001). Una Modesta Proposición [película]. Argentina

Pucciarelli, A. (2011). Menemismo. La construcción política del peronismo neoliberal. En A. Pucciarelli (coord.), Los años de Menem. La construcción del orden neoliberal. Buenos Aires: Siglo XXI.

Santillán, M. L. (2012). Quiénes educan a los chicos. Infancia, trayectorias educativas y desigualdad. Buenos Aires: Biblos.

Sirvent, M. T. (2008). Educación de adultos: investigación, participación, desafíos y contradicciones. Buenos Aires: Miño y Dávila editores.

Scarfó, G. (2016). Tesis doctoral inédita. Facultad de Filosofía y Letras, UBA, Buenos Aires.

Schulz, J. S. (2014). La transformación del Partido Justicialista, 1983-1995. Desindicalización, institucionalización, clientelismo y política territorial. Trabajo presentado en las VIII Jornadas de Sociología de la UNLP

Telam, (21-04-2015). Murió el dirigente social Alberto Morlachetti, el fundador del Hogar Pelota de Trapo. Telam. Recuperado de: http://www.telam.com.ar/notas/201504/102281-muerte-albertomorlachetti-asignacion-universal-por-hijo.html

Verdú, M. C. (2009). Represión en democracia. De la "primavera alfonsinista” al "gobierno de los derechos humanos”. Buenos Aires: Herramienta Ediciones

Villalta, C. (2005). ¿De quién son los niños pobres? Los debates sobre la tutela administrativa, judicial o caritativa en Buenos Aires de fin de siglo pasado. Derechos humanos, tribunales $y$ policías en Argentina y Brasil. Buenos Aires: Antropofagia.

Villalta, C. (2010). La administración de la infancia en debate. Entre tensiones y reconfiguraciones institucionales. Estudios en Antropología Social, 1(2) 81-99. 